\title{
Der akute Myokardinfarkt: Leitliniengerechte Therapie in der Präklinik
}

Markus Jäckel, Christoph Bode, Dawid Staudacher

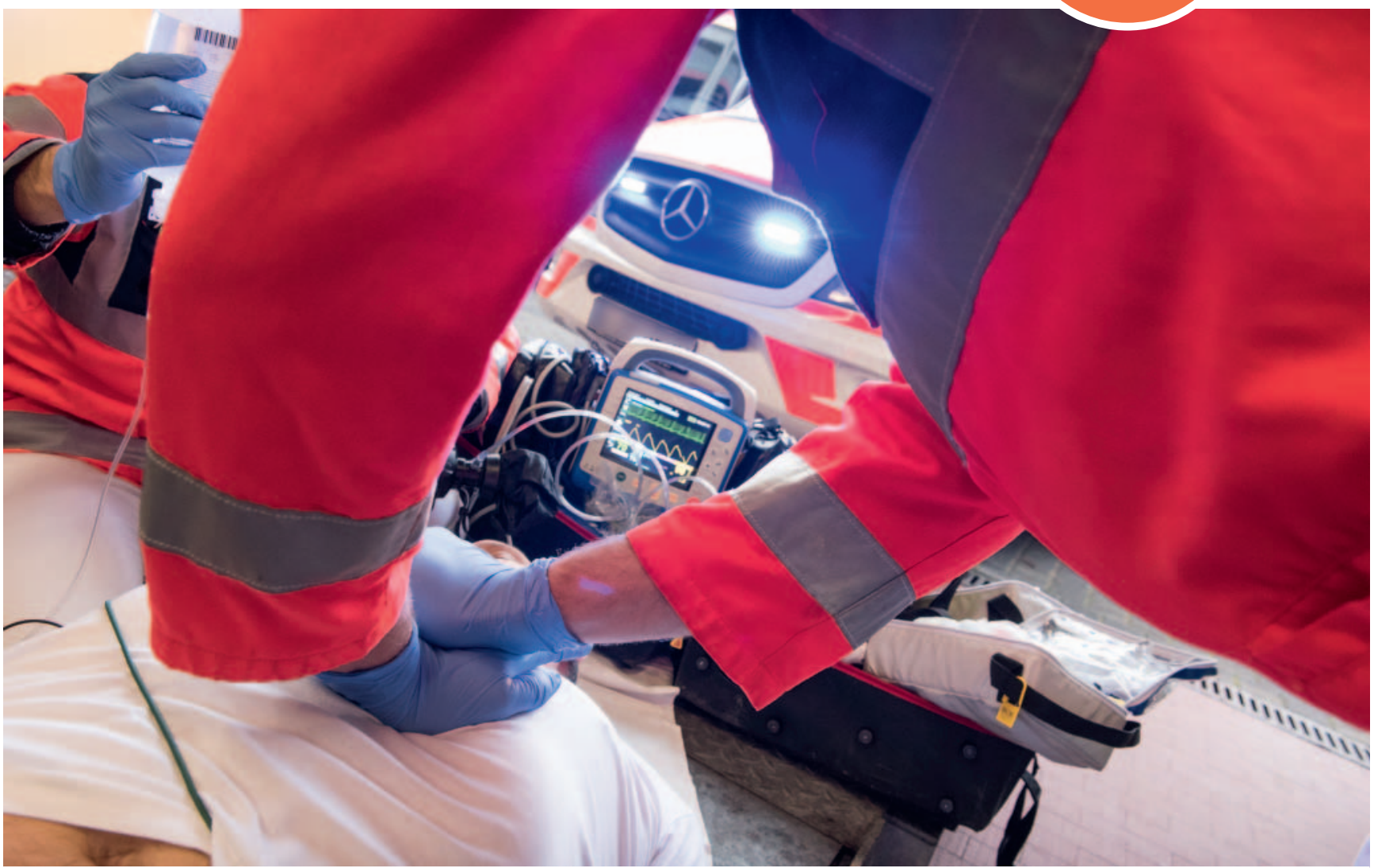

Quelle: KH Krauskopf (nachgestellte Situation)

Jährlich sterben in Deutschland über 40000 Menschen an den Folgen eines Myokardinfarkts. Unverändert versterben davon allerdings bis zu zwei Drittel präklinisch - was die Wichtigkeit der präklinischen Versorgung für Patienten mit Herzinfarkt unterstreicht. Dieser Beitrag will auf der Basis auf den aktuellen Leitlinien einen Überblick über präklinisch relevante Aspekte geben [1, 4, 7].

\section{Merke}

Direkt nach Entwicklung eines Herzinfarkts ist die Situation am kritischsten.

\section{Definition und Ätiologie}

Der Myokardinfarkt (oder Herzinfarkt; engl.: Acute Myocardial Infarction) ist als akute Minderdurchblutung des Herzens (Myokard, Herzmuskelgewebe) mit daraus folgendem Absterben von Herzmuskelgewebe definiert. Ätiologisch wird der Myokardinfarkt in 5 Typen eingeteilt, von denen nur die Typen 1-3 präklinisch relevant sind ( $\triangleright$ Tab. 1) [1].
Atherosklerose ist bei Weitem die häufigste Ursache des Myokardinfarkts, die am Herzen als koronare Herzkrankheit (KHK) bezeichnet wird. Die Atherosklerose führt durch Verkalkung der herzversorgenden Arterien zu Gefäßengen (Plaques). Wenn die Plaques aufbrechen und daraus folgend einen kompletten Verschluss der Herzarterie verursachen, führt dies zu einem Myokardinfarkt. Der Typ-2-Myokardinfarkt, z. B. im Rahmen einer hypertensiven Krise, ist ebenfalls häufig. 


\section{Die Arbeitsdiagnose ACS}

Unter die Arbeitsdiagnose akutes Koronarsyndrom (engl.: Acute Coronary Syndrome, ACS) fallen 3 Entitäten

( $\triangleright$ Tab. 2):

- STEMI (ST-Elevation Myocardial Infarction)

- NSTEMI (Non-ST-Elevation Myocardial Infarction)

- instabile Angina pectoris (iAP)

\section{Klinik}

Hauptsymptom des akuten Myokardinfarkts ist die Angina pectoris (AP, lateinisch für „enge Brust“). Als „typische“ Angina pectoris wird ein Engegefühl hinter dem Brustbein (retrosternal) mit Ausstrahlung in den linken Arm (seltener beide Arme, Hals oder Kinn) bezeichnet. Begleitende Symptome beim Herzinfarkt können unter anderem Luftnot, Übelkeit, Kaltschweißigkeit, Bauchschmerzen oder Synkopen sein [1]. Eher atypische Symptome für einen Myokardinfarkt sind unter anderem ein reißender oder stechender Schmerz (,wie ein Messerstich“), isolierte Luftnot oder eine Atemabhängigkeit der Schmerzen. Es präsentieren sich bis zu 30 \% der Frauen mit Myokardinfarkt ohne oder mit atypischen Beschwerden [2], was die Diagnostik des Herzinfarkts in der Präklinik bei Frauen erschwert. Auch ältere Patienten, Patienten mit chronischer Nierenerkrankung, Diabetes oder Demenz leiden häufig nur unter atypischen Beschwerden und bedürfen eines besonderen Augenmerks [3]. Somit kann kein klinisches Symptom einen Myokardinfarkt ausschließen oder beweisen. Eine sichere Diagnose des Myokardinfarkts ist erst in der Klinik nach ausführlicher Diagnostik möglich (s. u.).

\section{Merke}

Frauen mit Herzinfarkt stellen sich häufig ohne die typischen Symptome wie retrosternales Engegefühl mit Ausstrahlung in den linken Arm vor.

\section{Diagnostik}

\section{Anamnese und Untersuchung}

\section{FALLBEISPIEL}

Teil 1

Ein Rettungswagen wird notfallmäßig zu einem Einsatz (Stichwort „Übelkeit“) gerufen. Bei Eintreffen am Einsatzort findet das Einsatzteam einen kardiorespiratorisch stabilen adipösen 62-jährigen Patienten (AF 15/min, HF 100/min, RR 160/80 mmHg) vor. Er gibt an, dass er vorhin plötzlich unter starkem Erbrechen gelitten habe, außerdem sei ihm der Schweiß ausgebrochen, es sei mittlerweile jedoch wieder besser. Bis auf Probleme mit dem Blutzucker sei es ihm bisher immer gut gegangen. Der verantwortliche Notfallsanitäter entschließt sich zur Durchführung eines 12-Kanal-EKGs.

- Tab. 1 Klassifikation des Myokardinfarkts.

\begin{tabular}{|l|l|}
\hline Typ & Beschreibung \\
\hline 1 & $\begin{array}{l}\text { Ruptur einer atherosklerotischen Plaque, Einreißung oder Dissek- } \\
\text { tion eines Koronargefäßes mit konsekutiver Myokardischämie }\end{array}$ \\
\hline 2 & $\begin{array}{l}\text { Sauerstoffminderversorgung des Myokards infolge relativer } \\
\text { Minderdurchblutung, z. B. bei Tachyarrhythmie, Bradykardie, } \\
\text { Koronarspasmen, Hypotonie, Hypertonie }\end{array}$ \\
\hline 3 & $\begin{array}{l}\text { hochgradiger Verdacht auf Myokardinfarkt (z. B. ST-Hebungen) } \\
\text { mit Tod vor definitiver Diagnostik }\end{array}$ \\
\hline 4 & Myokardinfarkt während/nach Herzkatheteruntersuchung \\
\hline 5 & Myokardinfarkt während/nach Bypass-Operation \\
\hline
\end{tabular}

- Tab. 2 Einteilung des akuten Koronarsyndroms.

\section{Leitsymptom: akuter Thoraxschmerz}

\begin{tabular}{|l|l|l|l|}
\hline Arbeitsdiagnose & ACS & ACS & ACS \\
\hline Angina pectoris & ja & ja & ja \\
\hline ST-Hebungen im EKG & ja & nein & nein \\
\hline Synonym & STE-ACS & NSTE-ACS & NSTE-ACS \\
\hline Troponin T initial & negativ & negativ & negativ \\
\hline Troponin T 3 h nach Beschwerden & deutlich angestiegen & deutlich angestiegen & gleichbleibend \\
\hline finale Diagnose & STEMI & NSTEMI & instabile Angina pectoris \\
\hline Herzkatheter & $<120$ min & nach Risikoeinschätzung & elektiv \\
\hline
\end{tabular}

ACS: Acute Coronary Syndrome; EKG: Elektrokardiografie; NSTE: Non-ST-Elevation; NSTEMI: Non-ST-Elevation Myocardial Infarction; STE: ST-Elevation; STEMI: ST-Elevation Myocardial Infarction. 
- Tab. 3 Differenzialdiagnosen des Myokardinfarkts.

\begin{tabular}{|c|c|}
\hline Diagnose & Mögliche klinische Präsentation \\
\hline Aortendissektion & $\begin{array}{l}\text { stärkster, reißender Thoraxschmerz, evtl. Blut- } \\
\text { druckseitendifferenz, bekannte schwere art. } \\
\text { Hypertonie, bekannte bikuspide Aortenklappe }\end{array}$ \\
\hline Perikarditis & $\begin{array}{l}\text { stechender retrosternaler Schmerz, verstärkt im } \\
\text { Liegen/Inspiration, konkave ST-Hebungen ohne } \\
\text { spez. Lokalisation }\end{array}$ \\
\hline Lungenembolie & $\begin{array}{l}\text { Dyspnoe, Tachykardie, Thoraxschmerz, Hämoptysen, } \\
\text { Reiseanamnese, Umfangsvermehrung der Beine }\end{array}$ \\
\hline $\begin{array}{l}\text { (Spannungs-) } \\
\text { Pneumothorax }\end{array}$ & Anamnese, Dyspnoe, Einflussstauung, Auskultation \\
\hline Ösophagusruptur & $\begin{array}{l}\text { Vernichtungsschmerz, meist nach Erbrechen } \\
\text { beginnend, Dyspnoe, evtl. Hämatemesis, Haut- } \\
\text { emphysem }\end{array}$ \\
\hline Pankreatitis & $\begin{array}{l}\text { gürtelförmiger epigastrischer Schmerz, Übelkeit, } \\
\text { Erbrechen }\end{array}$ \\
\hline Gallenkolik & $\begin{array}{l}\text { kolikartiger Schmerz, primär im rechten Oberbauch, } \\
\text { gelegentlich Ausstrahlung in rechte Schulter }\end{array}$ \\
\hline
\end{tabular}

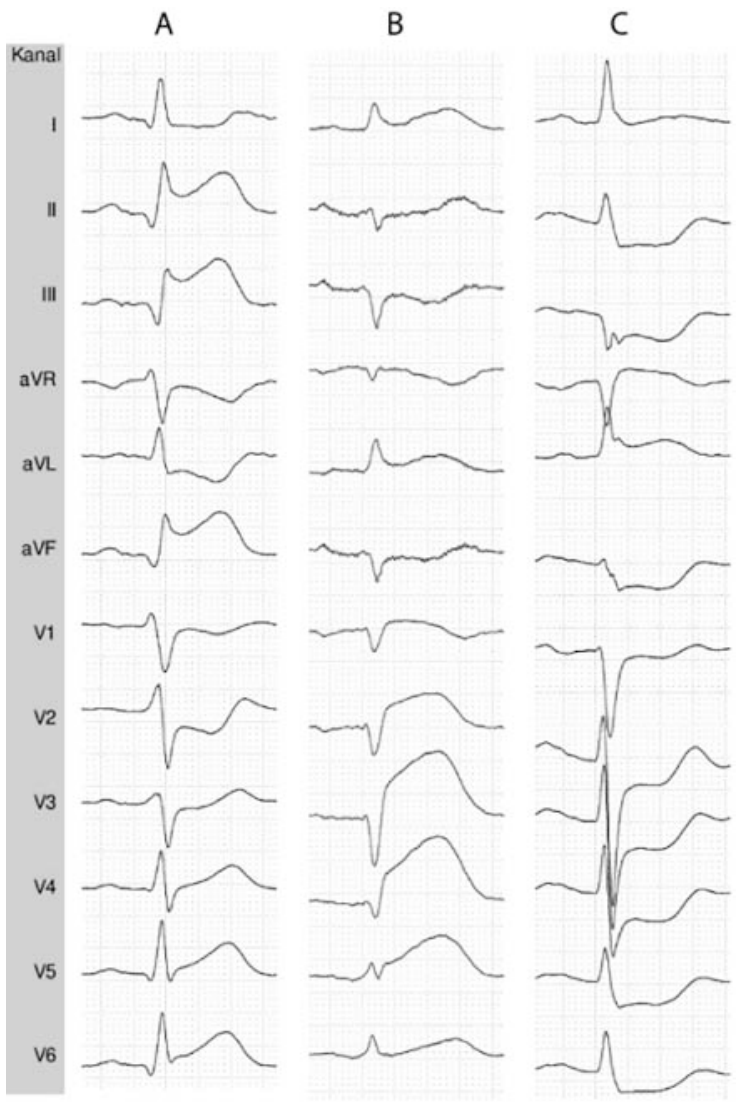

- Abb. 1 12-Kanal-EKG mit STEMI. A: Hinterwandinfarkt; B: Vorderwandinfarkt; C: STEMI-äquivalent: ST-Senkungen $>1 \mathrm{~mm}$ in 8 oder mehr Ableitungen $+\mathrm{ST}$-Hebung in aVR.
Bei Erstkontakt mit dem Patienten sollte eine gezielte Anamnese erfolgen und besonders nach herzinfarkttypischen Symptomen (s. u.) gefragt werden. Das Erfragen der kardiovaskulären Risikofaktoren (siehe folgende Übersicht) oder typischer Medikamente kann helfen, Risikopatienten zu identifizieren. Eine gezielte körperliche Untersuchung, auch zur Differenzialdiagnostik, insbesondere Aortendissektion, Lungenarterienembolie, Spannungspneumothorax und Ösophagusperforation (für eine unvollständige Übersicht s. > Tab. 3), ist unerlässlich.

\section{ÜBERSICHT}

\section{Kardiovaskuläre Risikofaktoren}

- männliches Geschlecht

- positive Familienanamnese (koronare Herzerkrankung bei Familienangehörigen 1. Grades vor dem 55. (männlich) bzw. 65. (weiblich) Lebensjahr)

- Nikotinkonsum

- Diabetes mellitus

- Hypercholesterinämie

- Adipositas

\section{EKG}

\section{FALLBEISPIEL}

Teil 2

Es zeigt sich das in > Abb. 1, A, dargestellte EKG. Bei STEMI wird der Notarzt nachgefordert, ein venöser Zugang sowie eine kontinuierliche Monitorüberwachung werden etabliert. Der Notarzt ist rasch vor Ort. Es erfolgt die Gabe von 300 mg ASS + 5000 IE Heparin i.v. Mittlerweile klagt der Patient über progredienten Thoraxschmerz, sodass eine fraktionierte Gabe von Morphin (kumulativ $5 \mathrm{mg}$ i. v.) sowie eines Antiemetikums erfolgt. Parallel dazu telefoniert der Notarzt bereits mit der nächsten Klinik mit Herzkatheterlabor und vereinbart, diese direkt anzufahren.

Die Differenzierung zwischen STEMI und NSTEMI/iAP hat größte Relevanz für die präklinische Versorgung, da beim STEMI jede Minute zählt.

\section{Merke}

Das EKG ist das wichtigste Werkzeug bei dem Verdacht auf einen Myokardinfarkt.

Zusätzlich kann das initiale EKG-Monitoring lebensbedrohliche Arrhythmien identifizieren, weswegen dieses umgehend etabliert und bis zur Patientenübergabe in der Klinik erfolgen sollte [4]. Laut Leitlinie darf die Zeit 
zwischen medizinischem Erstkontakt und Befundung des 12-Kanal-EKGs maximal 10 min betragen $[4,5]$.

\section{Merke \\ Ein 12-Kanal-EKG sollte bei Verdacht auf einen Myo- kardinfarkt innerhalb von 10 min nach Eintreffen des Rettungsdienstes geschrieben und interpretiert sein.}

Liegt eine entsprechende Klinik vor (und nur dann), wird ein STEMI anhand der in der folgenden Übersicht genannten EKG-Kriterien diagnostiziert ( $>$ Abb. 1) [4]. EKGVeränderungen sind häufig dynamisch und treten manchmal erst ca. 20 min nach Symptombeginn auf (initial zeigt sich dann oft eine T-Überhöhung, das „Erstickungs-T“). Dann können sequenzielle EKG-Kontrollen sinnvoll sein [4]. Eine telemetrische Übertragung des EKGs an die Zielklinik kann hilfreich sein. Eine initiale Labordiagnostik wird präklinisch nicht empfohlen.

\section{ÜBERSICHT}

\section{EKG-Kriterien eines STEMI}

- ST-Hebung in 2 angrenzenden Ableitungen: V2V3 $>2,5 \mathrm{~mm}$ ( $\mathrm{m}<40$ Jahre), $>2 \mathrm{~mm}$ ( $\mathrm{m}>40$ Jahre), $>1,5 \mathrm{~mm}(\mathrm{w})$ und/oder $>1 \mathrm{~mm}$ in anderen Ableitungen ohne Nachweis eines Linksschenkelblocks (LSB); (10 mm entsprechen $1 \mathrm{mV}$ )

- Bei inferiorem Infarkt sollten rechts präkordiale Ableitungen (V3 R und V4 R) zur Diagnostik eines rechtsventrikulären Infarkts erfolgen.

- Bei ST-Senkungen in V1-V3 sollten posteriore Ableitungen (V7-V9) erfolgen, Hebungen $>0,5 \mathrm{~mm}$ bestätigen einen posterioren STEMI.

- Bei LSB sind konkordante ST-Hebungen gute Indikatoren für einen STEMI (Sgarbossa-Kriterien).

- Patienten mit LSB und klinischer Symptomatik sollten als STEMI-äquivalent behandelt werden, unabhängig davon, ob der LSB bekannt ist.

- Bei Patienten mit Herzschrittmacher mit ventrikulärer Stimulation oder bei Patienten mit Rechtsschenkelblock sollte eine Behandlung als STEMI-äquivalent erwogen werden.

- ST-Senkungen $>1 \mathrm{~mm}$ in 8 oder mehr Ableitungen + ST-Hebung in aVR und/oder V1 sollten ebenfalls als STEMI-äquivalent behandelt werden (V.a. Hauptstammstenose).

\section{Therapie}

\section{Abarbeiten nach $A B C D E-S c h e m a$}

Nach Eintreffen bei einem Patienten mit Verdacht auf Myokardinfarkt sollte eine fokussierte Erhebung des Patientenstatus nach dem ABCDE-Schema erfolgen (Airway, Breathing, Circulation, Disability, Environment,
Exposure). Erst wenn sich nach Abarbeitung des ABCDESchemas keine lebensbedrohlichen Diagnosen ergeben oder diese adressiert wurden, wird die spezifische Diagnostik des Myokardinfarkts durchgeführt.

\section{Merke}

In den ersten 60-90 s nach Patientenkontakt sollte das $A B C D E-S c h e m a$ abgearbeitet werden. Ein engmaschiges Monitoring und die kontinuierliche Überwachung des Patienten sind obligat.

\section{Blutverdünnung}

Da bei einem Herzinfarkt die Blutversorgung meist durch einen Thrombus im Herzkranzgefäß unterbrochen ist, stellt die Blutverdünnung einen wichtigen Baustein der Therapie dar. Aktuelle Leitlinien empfehlen die Gabe sowohl von Acetylsalicylsäure (ASS, Aspirin ${ }^{\circledR}, 150-300$ mg intravenös) als auch von Heparin (UFH, 70-100 IE/kg KG, max. 5000 IE i. v.). Es konnte gezeigt werden, dass eine höhere Dosis von ASS, wie früher üblich, bei akutem Koronarsyndrom keinen Überlebensvorteil bringt [6]. Wichtig ist, dass vor Gabe der Medikamente auf Allergien und auf Kontraindikationen (wie Hirnblutung, kürzlich durchgemachter Schlaganfall oder aber große OP bzw. Magenblutung in der Anamnese) geachtet werden muss. Im Zweifelsfall muss die Gabe als Einzelfallentscheidung von dem behandelnden Arzt indiziert werden.

\section{Merke \\ Die Standardtherapie bei Patienten mit Verdacht auf einen Myokardinfarkt besteht aus ASS 150-300 mg i. v. und unfraktioniertem Heparin 5000 IE i. v.}

Bezüglich der Gabe des zweiten Thrombozytenaggregationshemmers ist die Datenlage nicht eindeutig, sodass von den aktuellen Leitlinien keine Empfehlung bezüglich des genauen Einnahmezeitpunkts gegeben wird [1, 4, 7]. Im Rahmen der Herzkatheteruntersuchung erfolgt aber immer eine duale Thrombozytenaggregationshemmung mit Aspirin und einem weiteren P2Y12-Hemmer (Clopidogrel, Ticagrelor, Prasugrel). Dabei sollten lokale Protokolle beachtet werden.

Ist eine heparininduzierte Thrombozytopenie (HIT) bekannt, wird Bivalirudin $(0,75 \mathrm{mg} / \mathrm{kg} \mathrm{KG}$ als Bolus empfohlen, gefolgt von einer Infusion mit 1,75 mg/kg KG/h).

\section{Kreislauf}

Manche Patienten mit Herzinfarkt präsentieren sich mit stark hypertensiven Blutdruckwerten, die einerseits häufig chronisch sind und andererseits durch Aufregung, Angst und Schmerzen bedingt sein können. Fraktionierte Opiat- und bei starker Angst auch Benzodiazepingabe sollten dann erwogen werden. Wenn nach Symptomlinderung weiterhin stark hypertensive Blutdruckwerte zu 
messen sind, empfehlen sich Nitrate (z. B. 2 Hübe Nitro s. I. bei einem systolischen Blutdruck $>180 \mathrm{mmHg}$.

Nitrate (Nitroglyzerin, TNS) werden von den aktuellen Leitlinien nur zur Symptomkontrolle empfohlen und haben keinen Einfluss auf das Überleben [8]. Relevante Kontraindikation (z. B. Einnahme von PDE-5-Hemmern) müssen beachtet werden. Eine Reduktion der Symptomatik nach Gabe von Nitroglyzerin kann zu Fehlinterpretationen führen, sodass dies nicht als Diagnostikmethode empfohlen wird.

Sind die Patienten nicht nur hypertensiv, sondern auch noch tachykard, kann eine vorsichtige Gabe eines intravenösen Betablockers (z. B. Metoprolol $1 \mathrm{mg}$ i. v. fraktioniert) erfolgen. Eine routinemäßige Gabe eines Betablockers, wie früher empfohlen, sollte nicht mehr durchgeführt werden.

\section{Merke}

Betablocker und Nitroglyzerin nur zur Behandlung einer deutlichen Hypertonie einsetzen.

\section{Schmerztherapie}

Angst und Schmerz führen zu Sympathikusaktivierung und damit zur Vasokonstriktion der peripheren Gefäße, was die Herzarbeit erhöht. Aus diesem Grund sowie zur Entspannung der Atmosphäre sind Angst- und Schmerztherapie essenziell. Am häufigsten wird Morphin verwendet. Auf eine zu liberale Gabe sollte jedoch verzichtet werden, da Morphin die Wirkung von Thrombozytenaggregationshemmern reduziert [9].

\section{Sauerstoff}

Früher war die Gabe von Sauerstoff bei Patienten mit Herzinfarkt Standard. Heute wissen wir aber, dass auch Sauerstoff als Medikament anzusehen ist, und eine Gabe eines Medikaments an Patienten, die es nicht benötigen, diesen schaden kann. So ergeben sich Hinweise, dass eine liberale Sauerstoffgabe die Myokardinfarktnarbe sogar vergrößern kann [10]. Sauerstoff sollte deshalb nur bei einer peripheren Sauerstoffsättigung von $<90 \%$ oder Symptomatik (Hypoxie, Luftnot, Herzinsuffizienzzeichen) gegeben werden (Zielsättigung 94-98\%, keinesfalls $100 \%)$.

\section{Merke}

Die Sauerstoffgabe gehört nicht mehr zur Standardtherapie des akuten Myokardinfarkts.

\section{Herzkatheteruntersuchung}

Da die Ursache des Herzinfarkts eine Minderdurchblutung des Herzens ist, ist die Wiedereröffnung der Herzkranzarterie die einzige kausale Therapie. Ziel sollte deshalb die rasche Reperfusion des minderdurchbluteten Myokards sein - was mittels perkutaner transluminaler Koronarangioplastie (PTCA) im Rahmen einer Herzkathe- teruntersuchung erfolgen kann. Dabei kommt das EKG ins Spiel. Zeigen sich ST-Hebungen im EKG, dann zählt jede Minute. Patienten müssen umgehend direkt ins nächste Herzkatheterlabor gebracht werden - ein Umweg über die Notaufnahme oder die Intensivstation kostet Menschenleben. Daher ist es sinnvoll, das medizinische Personal des Herzkatheterlabors frühzeitig zu informieren. Die aktuellen Leitlinien geben dazu eine Obergrenze von 120 min zwischen Diagnosestellung des STEMI (sollte innerhalb von 10 min nach medizinischem Erstkontakt erfolgen) und Durchführung der Herzkatheteruntersuchung an, wobei die kürzestmögliche Zeit angestrebt wird. Merke
STEMI = umgehende Anfahrt in ein Herzkatheterlabor
zur PTCA

Ein STEMI ist ein äußerst zeitkritischer Notfall; daher sollte ein direkter Transport in ein Herzkatheterlabor erfolgen.

\section{Lysetherapie}

In Einzelfällen gelingt es nicht, die Herzkatheteruntersuchung innerhalb von 120 min durchzuführen (z. B. bei widrigem Wetter). Sollte dieser Sonderfall eintreten und sollten die Beschwerden des Patienten sicher $<12 \mathrm{~h}$ bestehen, wird, sofern nicht kontraindiziert, eine intravenöse fibrinolytische Therapie (Lysetherapie) empfohlen [4]. Wichtig zu verstehen ist, dass die Lysetherapie besonders gut bei frischen Thromben wirkt, während die Blutungsgefahr (bis hin zu tödlichen Hirnblutungen) unabhängig von der Beschaffenheit des Thrombus in der Herzkranzarterie ist. Aktuelle Leitlinien sehen für die Indikationsstellung der Lysetherapie die ersten 10 min nach Diagnosestellung STEMI vor. In beiden Fällen sollte ein Zentrum mit Herzkatheter angefahren werden, da auch Patienten mit primärer Lysetherapie eine zeitnahe Herzkatheteruntersuchung erhalten sollten.

Merke

STEMI und Transportzeit und Herzkatheterlabor $>120 \mathrm{~min}$. Sofortige Lyse, sofern nicht kontraindiziert.

\section{Transport: Wohin mit Patienten ohne ST-Hebungen im EKG?}

Oft lässt sich präklinisch ein Myokardinfarkt nicht sicher diagnostizieren, wenn sich im EKG kein STEMI zeigt. Diese Patienten müssen aber natürlich dennoch in ein Akutkrankenhaus aufgenommen und weiter abgeklärt werden. Aktuelle Leitlinien empfehlen in solchen Fällen eine Risikostratifizierung der Patienten mit Verdacht auf ACS ( Abb. 2). 


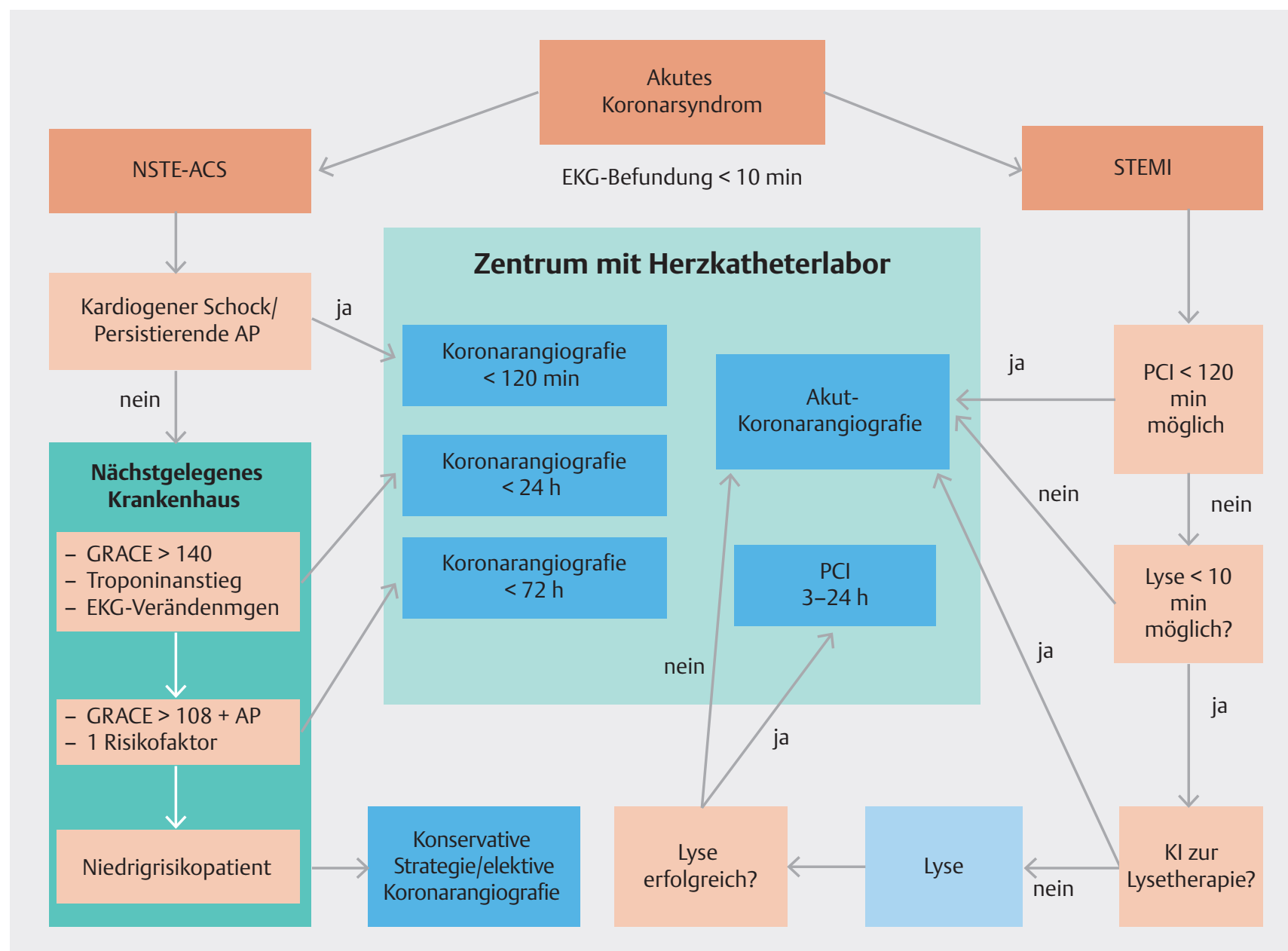

- Abb.2 Evaluation des Transportziels bei Patienten mit akutem Koronarsyndrom. ACS: Acute Coronary Syndrome; AP: Angina pectoris; EKG: Elektrokardiografie; GRACE: Global Registry of Acute Coronary Events (Punktwert zur Risikoabschätzung: siehe Internet); KI: Kontraindikation; NSTE: Non-ST-Elevation; PCI: Perkutane koronare Intervention; STE: ST-Elevation; STEMI: ST-Elevation Myocardial Infarction.

\section{Komplikationsmanagement}

\section{FALLBEISPIEL}

Teil 3

Kurz nach Transportbeginn verdreht der Patient plötzlich die Augen und verliert das Bewusstsein, ein rascher Blick auf den Monitor zeigt eine HF von 180/min mit breiten QRS-Komplexen, das Einsatzteam diagnostiziert eine VT und kardiovertiert den Patienten, was primär erfolgreich ist und zu raschem Wiedererlangen des Bewusstseins führt. Nach ca. 5 min kommt es zu einer erneuten VT, diesmal jedoch ohne hämodynamische Relevanz. Der Notarzt entschließt sich zur Gabe von $300 \mathrm{mg}$ Amiodaron in $100 \mathrm{ml}$ G5\% als Kurzinfusion über 20-60 min. Nach erneut ca. 5 min kommt es zur Konversion in einen stabilen Sinusrhythmus. Im Anschluss ist der Patient kardiorespiratorisch stabil.
Komplikationen im Rahmen eines akuten Myokardinfarkts sind häufig und individuell verschieden. Dazu kann nur ein kurzer, unvollständiger Überblick gegeben werden.

Der kardiogene Schock wird definiert als systolischer Blutdruck $<90 \mathrm{mmHg}$ mit Hypoperfusionszeichen und ist eine häufige Komplikation des Myokardinfarkts. Primäres Ziel sollte der schnellstmögliche Transport ins Herzkatheterlabor zur Ursachenbehandlung sein. Sofern es nicht bereits zum Rückwärtsversagen mit Lungenödem gekommen ist (ggf. auch dann), kann ein Volumenversuch erfolgen. Zur Aufrechterhaltung des Kreislaufs muss, falls notwendig, eine Katecholamintherapie erfolgen (Dobutamin/Noradrenalin).

Deutlich häufiger als der kardiogene Schock sind ventrikuläre Arrhythmien (ventrikuläre Tachykardie, VT, und Kammerflimmern, VF), die auch Haupttodesursache beim akuten Myokardinfarkt sind [11]. Aus diesem Grund muss immer eine Reanimationsbereitschaft bestehen. 
Auch dann gilt es eine schnellstmögliche Ursachenbehandlung, d. h. Reperfusion, anzustreben. Liegt eine VT vor, kann bei fehlender hämodynamischer Relevanz Amiodaron gegeben werden (z. B. $300 \mathrm{mg}$ Amiodaron in Glukose $5 \% 100 \mathrm{ml}$ als Kurzinfusion über 20-60 min). Bei hämodynamischer Relevanz muss kardiovertiert werden. Bei Bradykardien mit hämodynamischer Relevanz kann ein medikamentöser Therapieversuch (z. B. Adrenalin, Atropin) erfolgen, ggf. muss extern stimuliert werden.

Bei Patienten mit Kreislaufstillstand erfolgt die kardiopulmonale Reanimation entsprechend den aktuellen Leitlinien [12]. Nach ROSC (Return of Spontaneous Circulation) muss umgehend ein 12-Kanal-EKG geschrieben werden. Bei STEMI sollte das Herzkatheterlabor als Zielstruktur angefahren werden. Bei sonstigem hochgradigem Verdacht auf einen ursächlichen Myokardinfarkt (z. B. zuvor angegebener Brustschmerz, bekannter KHK, erster Rhythmus VF) sollte das Herzkatheterlabor als Zielstruktur erwogen werden [4].

\section{Innerklinisches Prozedere}

\section{FALLBEISPIEL}

Teil 4

Im Herzkatheterlabor zeigt sich der in $\mathbf{A b b} \mathbf{3}$ gezeigte Befund. Nach PCI wird der Patient auf die Intensivstation gebracht. Der postinterventionelle Verlauf gestaltet sich komplikationslos und der Patient kann nach 5 Tagen entlassen werden.

Nach Erreichen der Zielstruktur wird der Patient an das dortige Behandlungsteam übergeben. Nach der Herzkatheteruntersuchung ( $\boldsymbol{A}$ Abb. $\mathbf{3}$ ) wird der Patient üblicherweise auf der Intensivstation überwacht. Die weitere stationäre Therapie gilt der Überwachung von Herzrhythmusstörungen, der medikamentösen Therapie einer ggf. auftretenden Herzinsuffizienz und der Einstellung der kardiovaskulären Risikofaktoren. Die typische Krankenhausverweildauer von Patienten mit Herzinfarkt beträgt 3-7 Tage. Eine Anschlussheilbehandlung für mehrere Wochen wird nach Entlassung empfohlen.

\section{KERNAUSSAGEN}

- Die präklinische Mortalität beim akuten Myokardinfarkt ist weiterhin hoch.

- Hauptsymptom des akuten Myokardinfarkts ist die Angina pectoris. Insbesondere bei Frauen, Diabetikern und älteren Patienten ist die klinische Präsentation jedoch häufig atypisch.

- Zur Diagnostik muss ein 12-Kanal-EKG erfolgen. Aufgrund von lebensbedrohlichen Arrhythmien
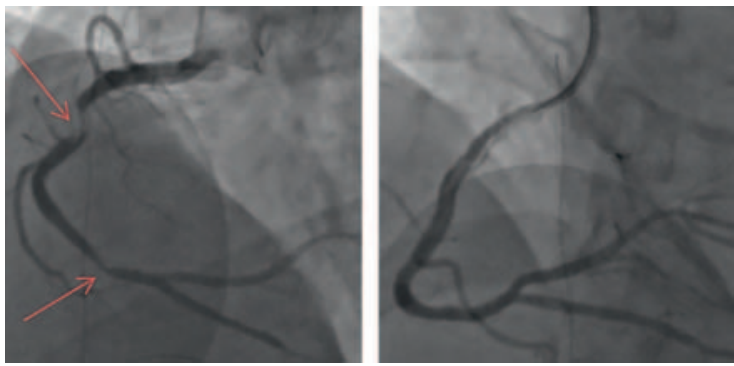

- Abb. 3 Herzkatheterbefund bei einem Patienten mit STEMI der Hinterwand und hochgradigen Stenosen der rechten Koronararterie (Pfeile) vor (links) und nach PCI (rechts).

muss ein kontinuierliches EKG-Monitoring durchgeführt werden.

- Die Auswahl der richtigen Zielklinik und die damit verbundenen lokalen Protokolle sollten jedem Rettungsdienstmitarbeiter und Notarzt bekannt sein.

- Aspirin und Heparin sind die einzigen therapeutisch wichtigen präklinischen Medikamente. Sauerstoff verbessert die Prognose nicht und sollte nur bei Hypoxie, Luftnot und Herzinsuffizienzsymptomatik gegeben werden.

- Haupttodesursache sind Arrhythmien wie Kammerflimmern und ventrikuläre Tachykardien. Eine andere häufige Komplikation ist der kardiogene Schock mit Vorwärts- und/oder Rückwärtsversagen.

\section{Interessenkonflikt}

Die Autorinnen/Autoren geben an, dass kein Interessenkonflikt besteht.

\section{Autorinnen/Autoren}

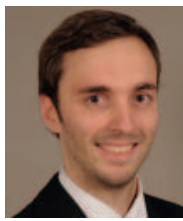

\section{Markus Jäckel}

Dr., 2006-2007 Rettungssanitäterausbildung

i. R. FS] in Lindau. 2007-2013 Studium der Medizin in Innsbruck. 2014-2019 Facharztausbildung Innere Medizin (Kardiologie I, Prof. Bode, Freiburg). 2019 Facharzt Innere Medizin. 2020 Zusatzbezeichnung Notfallmedizin Schwerpunkt: Internistische Intensivmedizin.

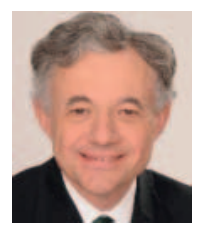

\section{Christoph Bode}

Prof. Dr. Dr. h. c., 1974-1980 Studium der Medizin in Köln. 1983-1992 Facharztausbildung Universität Heidelberg. 1992-1999 Oberarzt. Seit 1999 Ordinarius Kardiologie und Angiologie der Universität Freiburg. Seit 2012 ärztlicher Direktor der Klinik für internistische Intensivmedizin des Universitätsklinikums Freiburg und der Klinik für Kardiologie und Angiologie I des Universitäts-Herzzentrums Freiburg - Bad Krozingen. 


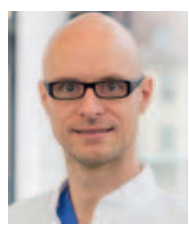

\section{Dawid Staudacher}

Dr., 1999-2005 Studium der Humanmedizin in Wien und Alicante. Nach einem Forschungsaufenthalt in Haifa seit 2006 an der Uniklinik Freiburg tätig. Rettungssanitäter (1998), Notarzt (2011), Internist (2013), Intensivmediziner (2015) und Kardiologe (2017). Schwerpunkte: kardiologische Intensivmedizin und extrakorporaler Organersatz.

Korrespondenzadresse

\section{Dr. med. Markus Jäckel}

Klinik für Kardiologie und Angiologie I

Universitäts-Herzzentrum Freiburg Bad Krozingen

Hugstetter Straße 55

79106 Freiburg

Deutschland

markus.jaeckel@universitaets-herzzentrum.de

\section{Literatur}

[1] Roffi M, Patrono C, Collet JP et al. 2015 ESC Guidelines for the Management of Acute Coronary Syndromes in Patients Presenting Without Persistent ST-segment Elevation. Rev Esp Cardiol (Engl Ed) 2015; 68 (12): 1125. doi:10.1016/j.rec.2015.10.009

[2] Brieger D, Eagle KA, Goodman SG et al. Acute coronary syndromes without chest pain, an underdiagnosed and undertreated high-risk group: insights from the Global Registry of Acute Coronary Events. Chest 2004; 126 (2): 461-469. doi:10.1378/ chest.126.2.461

[3] Canto JG, Fincher C, Kiefe Cl et al. Atypical presentations among Medicare beneficiaries with unstable angina pectoris. Am J Cardiol 2002; 90 (3): 248-253. doi:10.1016| s0002-9149(02)02463-3

[4] Ibánez B, James S, Agewall S et al. 2017 ESC Guidelines for the management of acute myocardial infarction in patients presenting with ST-segment elevation. Rev Esp Cardiol (Engl Ed) 2017; 70 (12): 1082. doi:10.1016/j.rec.2017.11.010

[5] Tubaro M, Danchin N, Goldstein P et al. Pre-hospital treatment of STEMI patients. A scientific statement of the Working Group Acute Cardiac Care of the European Society of Cardiology. Acute Card Care 2011; 13 (2): 56-67. doi:10.3109/ 17482941.2011 .581292
[6] Mehta SR, Bassand JP, Chrolavicius S et al. Dose comparisons of clopidogrel and aspirin in acute coronary syndromes. New Engl J Med 2010; 363 (10): 930-942. doi:10.1056/ NEJMoa0909475

[7] Nikolaou NI, Arntz HR, Bellou A et al. European Resuscitation Council Guidelines for Resuscitation 2015 Section 8. Initial management of acute coronary syndromes. Resuscitation 2015; 95: 264-277. doi:10.1016/j.resuscitation.2015.07.030

[8] Borzak S, Cannon CP, Kraft PL et al. Effects of prior aspirin and anti-ischemic therapy on outcome of patients with unstable angina. TIMI 7 Investigators. Thrombin Inhibition in Myocardial Ischemia. Am J Cardiol 1998; 81 (6): 678-681. doi:10.1016 s0002-9149(97)01006-0

[9] Parodi G, Bellandi B, Xanthopoulou I et al. Morphine is associated with a delayed activity of oral antiplatelet agents in patients with ST-elevation acute myocardial infarction undergoing primary percutaneous coronary intervention. Circ Cardiovasc Interv 2015; 8 (1). doi:10.1161/ CIRCINTERVENTIONS. 114.001593

[10] Stub D, Smith K, Bernard S et al. Air Versus Oxygen in ST-Segment-Elevation Myocardial Infarction. Circulation 2015. 131 (24): 2143-2150. doi:10.1161/CIRCULATIONAHA.114.014494

[11] Larsen JM, Ravkilde J. Acute coronary angiography in patients resuscitated from out-of-hospital cardiac arrest - a systematic review and meta-analysis. Resuscitation 2012; 83 (12): 14271433. doi:10.1016/j.resuscitation.2012.08.337

[12] Monsieurs KG, Nolan JP, Bossaert LL et al. European Resuscitation Council Guidelines for Resuscitation 2015: Section 1. Executive summary. Resuscitation 2015; 95: 1-80. doi:10.1016/j.resuscitation.2015.07.038

\section{Bibliografie}

retten 2020; 9: 352-361

DOI 10.1055/a-1081-7676

ISSN 2193-2387

(c) 2020. Thieme. All rights reserved.

Georg Thieme Verlag KG, Rüdigerstraße 14,

70469 Stuttgart, Germany 


\section{Punkte sammeln auf CEEthieme.de}

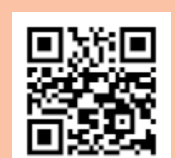

Diese Fortbildungseinheit ist in der Regel 12 Monate online für die Teilnahme verfügbar.

Den genauen Einsendeschluss finden Sie unter https://eref.thieme.de/CXED9W2.

Sollten Sie Fragen zur Online-Teilnahme haben, finden Sie unter https://cme.thieme.de/hilfe

eine ausführliche Anleitung. Wir wünschen viel Erfolg beim Beantworten

der Fragen!

Unter https://eref.thieme.de/CXED9W2 oder über den QR-Code kommen Sie

direkt zur Startseite des Wissenstests.

\section{Frage 1}

Beim Notarzteinsatz finden Sie einen 72-jährigen Patienten mit thorakalem Engegefühl vor. Im EKG sehen Sie folgenden Befund: HF 180/min, rhythmisch, schmale EKG-Komplexe, ST-Senkungen über der Vorderwand. Sie vermuten eine AV-Knoten-ReentryTachykardie und therapieren mit $6 \mathrm{mg}$ Adenosin. Es kommt zu einer Konversion in einen Sinusrhythmus, die EKG-Veränderungen sowie die Symptomatik des Patienten bilden sich rasch zurück. Um welchen Myokardinfarkt-Typ handelt es sich am wahrscheinlichsten?
A Typ 1
B Typ 2
C Typ 3
D Typ 4
E Typ 5

\section{Frage 2}

Ein 60-jähriger Patient $(90 \mathrm{~kg})$ mit folgendem klinischem Befund zeigt ST-Hebungen über der Hinterwand: starkes thorakales Engegefühl, Übelkeit, RR $120 / 80$ mmHg, SpO $294 \%$. Wie therapieren Sie präklinisch?

A ASS 150-300 mg i. v., Heparin 5000 IE, Morphin fraktioniert, $4 \mathrm{IO}_{2} / \mathrm{min}$ über die Nasenbrille

B ASS 500-1000 mg i.v., Heparin 5000 IE, Morphin fraktioniert, kein Sauerstoff

C ASS 150-300 mg i.v., Heparin 5000 IE, Morphin fraktioniert, kein Sauerstoff

D ASS 150-300 mg i.v. Heparin $5000 \mathrm{IE}, 15 \mathrm{I} \mathrm{O}_{2} / \mathrm{min}$ über eine Reservoir-Maske

E ASS 150-300 mg i.v., Rivaroxaban 20 mg, Morphin fraktioniert, $2 \mathrm{I} \mathrm{O}_{2} /$ min über die Nasenbrille

\section{Frage 3}

Ein Patient mit akutem Koronarsyndrom zeigt folgenden Befund im EKG: QRS-Komplex > 120 ms; breites R in V1/V2; tiefes, breites S in V5/V6. Der Patient hat eine bekannte KHK, leidet unter starker thorakaler Enge und ist kaltschweißig. Der Blutdruck liegt bei $80 / 50 \mathrm{mmHg}$, die Herzfrequenz bei $120 / \mathrm{min}$. Für welches Transportziel entscheiden Sie sich?

A direkte Anfahrt in ein Herzkatheterlabor

B Notaufnahme in einer Klinik mit Herzkatheter

C Hausarzt

D nächstes Krankenhaus (unabhängig ob Herzkatheter vorhanden)

E ambulante Versorgung des Patienten

\section{Frage 4}

Sie transportieren einen Patienten mit STEMI mit Symptomatik seit 1 Stunde. Aufgrund eines plötzlich einsetzenden Schneesturms verzögert sich der Transport um mehrere Stunden. Der Patient ist hämodynamisch stabil, Vorerkrankungen bestehen keine. Wie sollten Sie entsprechend der Leitlinie therapieren?

A Unverändert ist primäres Ziel weiterhin das Herzkatheterlabor.

B Lysetherapie präklinisch mit anschließendem Transport in die nächste Klinik, (unabhängig ob Herzkatheter vorhanden).

C Transportzieländerung auf das nächste Krankenhaus ohne Herzkatheterlabor (Transportzeit dorthin $30 \mathrm{~min}$ ). Dortige Lysetherapie.

D Lysetherapie präklinisch mit anschließendem Transport in die nächste Klinik mit Herzkatheterlabor.

E Lysetherapie mit anschließender Vorstellung beim Hausarzt.

- Weitere Fragen auf der folgenden Seite... 


\section{Punkte sammeln auf CEE.thieme.de}

\section{Frage 5}

Sie behandeln im Notarzteinsatz einen Patienten mit bekannter heparininduzierter Thrombozytopenie (HIT) und vermuten ein akutes Koronarsyndrom. Welche Therapie ist am geeignetsten?

A Heparin 5000 IE, eine HIT ist keine Kontraindikation im Notfall.

B Bivalirudin $(0,75 \mathrm{mg} / \mathrm{kg} \mathrm{KG}$ als Bolus, gefolgt von einer Infusion mit $1,75 \mathrm{mg} / \mathrm{kg} \mathrm{KG/h})$

C niedermolekularem Heparin s.c.

D neues orales Antikoagulans in therapeutischer Dosis (z. B. Rivaroxaban $20 \mathrm{mg}$ )

E ASS hochdosiert: $1000 \mathrm{mg}$

\section{Frage 6}

Welches der folgenden Medikamente muss beim akuten Koronarsyndrom (sofern nicht kontraindiziert) immer gegeben werden?
A ASS
B Morphin
C Nitrat
D Betablocker
E Lysetherapie

\section{Frage 7}

Welche Behauptung ist richtig?

A Haupttodesursache beim Myokardinfarkt ist der kardiogene Schock.

B Beim kardiogenen Schock sollte präklinisch immer ein extrakorporales Unterstützungsverfahren implantiert werden.

C Eine ventrikuläre Tachykardie mit hämodynamischer Relevanz sollte kardiovertiert werden.

D Bei Bradykardie mit Puls sollte eine Therapie mit Adrenalin (1 mg) erfolgen.

E Nach primär erfolgreicher Reanimation muss jeder Patient direkt ins Herzkatheterlabor transportiert werden.

\section{Frage 8}

Welches der folgenden EKG-Bilder kann nicht als STEMI-äquivalent behandelt werden?

A Linksschenkelblock
B Rechtsschenkelblock

C AV-Block Typ IIb

D ST-Senkungen $>1 \mathrm{~mm}$ in 8 oder mehr Ableitungen + ST-Hebung in aVR und/oder V1

E Herzschrittmacher mit ventrikulärer Stimulation

\section{Frage 9}

Bei welchen Patienten sind atypische Beschwerden beim Myokardinfarkt am seltensten?
A Frauen
B Diabetiker
C ältere Patienten
D demente Patienten
E sportliche Patienten

\section{Frage 10}

Was sollte nicht als Myokardinfarktdiagnostik durchgeführt werden?
A EKG
B Labordiagnostik
C "Nitro-Test"
D Vitalparameter
E Symptomatik des Patienten 